\title{
Gossypiboma en Neurocirugía. Descripción de un caso y revisión de la literatura
}

\author{
G. Chater-Cure; A. Fonnegra-Caballero; A.M. Baldión- Elorza* y E. Jiménez-Hakim
}

Departamentos de Neurocirugía y Patología*. Hospital Universitario Fundación Santa Fe de Bogotá. Colombia.

\section{Resumen}

Los términos gossypiboma, textiloma o cuerpo extraño textil retenido son términos médicos usados para describir elementos quirúrgicos textiles que intencional o accidentalmente son dejados sobre el lecho quirúrgico, generando una reacción a cuerpo extraño. Es una patología prevenible siempre y cuando se tomen algunas medidas durante la cirugía. Los gossypiboma son poco frecuentes a nivel del sistema nervioso central. Son más frecuentes en las cirugías torácicas o abdominales. Dependiendo de su localización y su comportamiento, éstos pueden presentar complicaciones y sintomatología o pueden permanecer silentes por muchos años.

En este trabajo se presenta un caso de un gossypiboma paraespinal asintomático en una paciente operada 15 años antes para una discectomía lumbar. Se hace además una revisión de la literatura y se comenta acerca de la implicación de estas lesiones en neurocirugía.

PALABRAS CLAVE: Textiloma. Gossypiboma. Reacción a cuerpo extraño. Masa paraespinal Sistema nervioso central. Neurocirugía.

Gossypiboma in Neurosurgery. Case report and literature review

\section{Summary}

The terms gossypiboma, textiloma or retained foreign objects are medical terms used to describe textile elements that are left intentionally or accidentally in the operative site and generate a reaction to foreign objects. It can be avoided when preventive measures are taken during surgery. Gossypibomas are rare in surgery of the central nervous system. They are more frequent in thoracic and abdominal surgeries. Depending on their location they can present with complica-

Recibido: 20-03-08. Aceptado: 19-08-08 tions and symptoms or they may remain undetected for many years.

In this article we want to present a case of a spinal asymptomatic gossypiboma in a patient who was operated on 15 years ago for a lumbar disectomy. We will review the literature on the implications of these lesions on the neurosurgical field.

KEY WORDS: Textiloma. Gossypiboma. Foreign object reaction. Spinal mass. Central nervous system. Neurosurgery.

\section{Introducción}

Los gossypiboma son poco frecuentes a nivel del sistema nervioso central. Son más frecuentes en las cirugías torácicas o abdominales. Un cuerpo extraño puede desencadenar una reacción inflamatoria que resulta en la formación de masas de gran tamaño. Los gossypibomas pueden causar muchos problemas incluyendo formación de abscesos, erosión del hueso adyacente, lesión vascular, obstrucción intestinal e infección de senos paranasales ${ }^{1}$.

Dependiendo de su localización en el sistema nervioso central y de su comportamiento, éstos pueden presentar complicaciones y sintomatología o pueden permanecer silentes por muchos años ${ }^{1,8,14}$. Se presenta un caso ilustrativo de este proceso tras una cirugía de columna lumbar.

\section{Caso clínico}

Se trata de una mujer de 41 años de edad a quien 15 años antes se le realizó una microdiscectomía lumbar L4L5 derecha por una hernia discal migrada, causante de una radiculopatía médicamente intratable. La paciente tuvo una excelente recuperación y permaneció asintomática hasta 4 meses antes de la consulta actual, cuando después de un accidente de tránsito se queja de dolor lumbar. Por lumbalgia persistente se le realizaron estudios imagenológicos donde incidentalmente se encontró una masa paraespinal derecha a nivel L4-L5 (Figuras 1-3). Se consideró que la lesión podría ser una masa de origen tumoral por sus características imagenológicas y su localización 


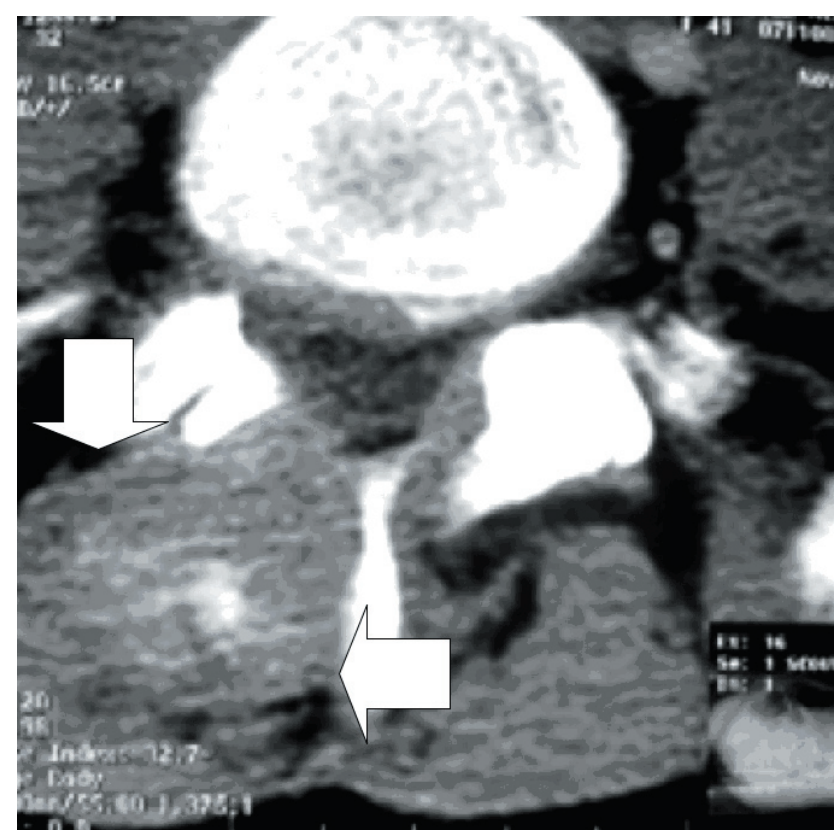

Figura 1. TAC de columna lumbar donde se puede ver una lesión redondeada, bien delimitada, paraespinal derecha (flechas).

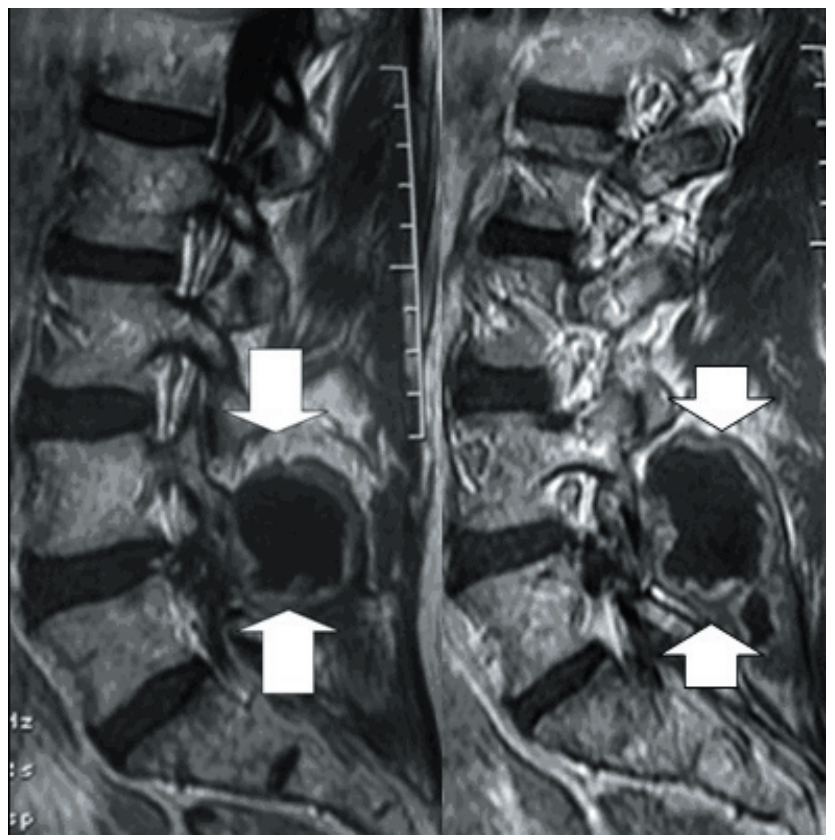

Figura 3. MRI corte sagital de un textiloma paraespinal. Llama la atención el centro hipo-intenso de la lesión y la capsula que lo circunscribe.
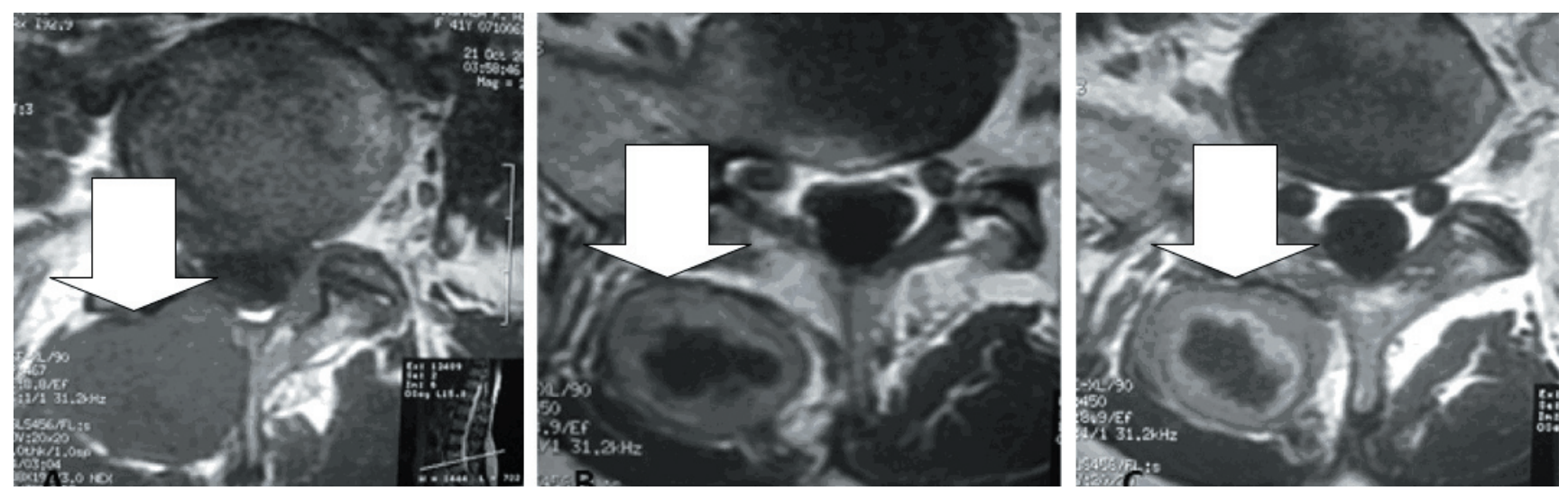

Figura 2. MRI de un gossypiboma paraespinal (flechas). Obsérvese: masa bien delimitada, con un componente externo (reacción inflamatoria crónica y encapsulamiento) y otro interno (el cuerpo extraño).

por lo cual se decidió llevar a la paciente a cirugía para resección de la lesión y estudio patológico de la misma. Inicialmente se pensó que la lesión podría corresponder a un rabdomiosarcoma, aunque llamaba la atención que correspondía exactamente al sitio de la cirugía previa. A la paciente se le practicó una resección en bloque de la masa lumbar. Se encontró una lesión encapsulada, fuertemente adherida a los tejidos vecinos sin embargo no tenía características infiltrantes. El procedimiento se realizó sin complicaciones, la recuperación fue completa y la paciente se reintegró a sus actividades a las pocas semanas.

\section{Patología}

Macroscópicamente la lesión correspondía a una masa ovoide de apariencia encapsulada que pesó 43 gramos y midió $7.5 \times 4.3 \mathrm{~cm}$. Externamente su superficie era lisa, de color rojizo y al corte de consistencia blanda $\mathrm{y}$ aspecto fibrilar con áreas granulares y amarillentas. Microscópicamente se observó con la coloración de hematoxilina eosina tejido fibroconectivo vascularizado con hemorragia, en el que se encuentra inmerso un cuerpo extraño a manera de fibras que en algunos focos eran de 


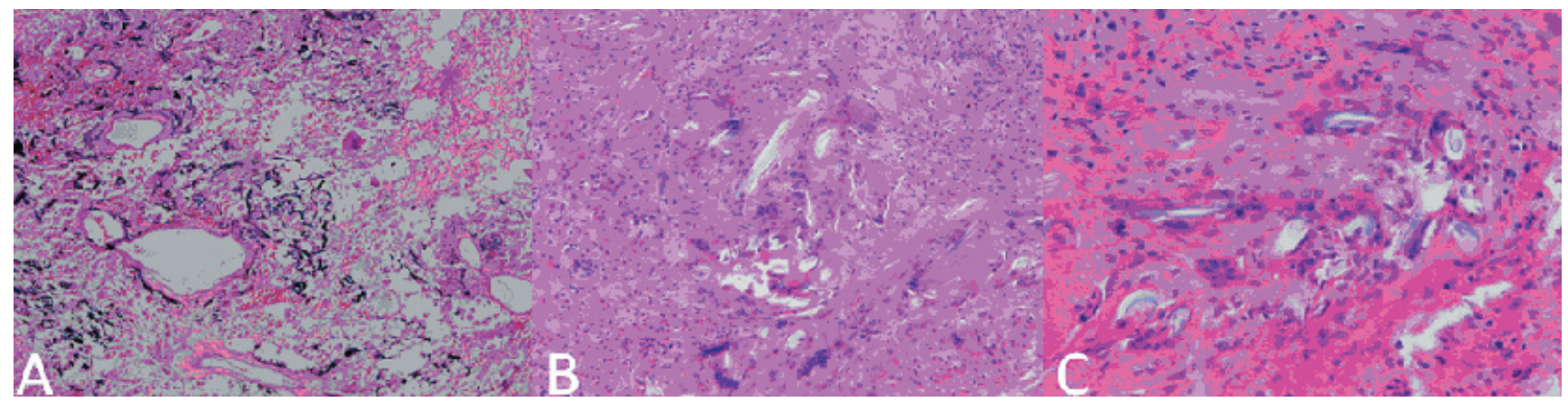

Figura 4. Coloración de hematoxilina eosina. A: tejido fibroconectivo vascularizado con hemorragia, en el que se encuentra inmerso material extraño a manera de fibras que en algunos focos son de color pardo oscuro. B(10x), C (40x): se observa reacción granulomatosa con células gigantes de tipo cuerpo extraño e inflamación crónica ligera rodeando cuerpo extraño.

color pardo oscuro y en otras blanco brillante. Rodeaba a esta lesión una reacción granulomatosa con células gigantes. Esto fue compatible con un cuerpo extraño, reacción granulomatosa e inflamación crónica ligera. (Figura 4).

En conclusión, se trata de un caso de gossypiboma que había estado silente por 15 años y fue diagnosticado incidentalmente en estudios imagenológicos por la lumbalgia mecánica secundaria al trauma que presentó la paciente.

\section{Discusión}

El termino gossypiboma es derivado del latín (Gossypium - algodón) y Kiswahili (Homa - lugar de encarcelamiento). Es una patología rara y poco informada en la literatura médica. Una razón para esto puede ser las implicaciones médico legales que tiene un evento de estos $^{13,15,17}$.

La incidencia de un material extraño retenido después de cirugía varía entre el $0.001 \%$ al $0.01 \%{ }^{15,16}$. Esta patología es más común a nivel abdominal, en las extremidades o a nivel torácico pero también ha sido informada en neurocirugía ${ }^{1,8,14}$.

Un cuerpo extraño puede desencadenar una reacción inflamatoria que resulta en la formación de masas de gran tamaño. Dependiendo de su localización, puede causar muchos problemas incluyendo formación de abscesos, erosión del hueso adyacente, lesión vascular, obstrucción intestinal e infección de senos paranasales ${ }^{1}$.

La presentación clínica puede ser aguda o crónica ${ }^{1}$. En la forma aguda o necrotizante predomina la reacción exudativa con formación de abscesos y fístulas cutáneas, y en la forma crónica hay encapsulación del cuerpo extraño formando un granuloma aséptico generalmente asintomático ${ }^{10,17}$.

En neurocirugía la manifestación clínica depende de su localización, siendo en el SNC los gossypibomas espinales los más frecuentes, y de evolución crónica. A nivel intracraneal la presentación suele hacerse en forma aguda $^{1,11}$.

\section{Gossypiboma Intracraneal}

A nivel intracraneal el término gossypiboma se refiere a material textil como cotonoides, material reabsorbible dejado de manera accidental o intencional para la realización de hemostasia ${ }^{11,14}$.

La presentación clínica de los gossypibomas intracraneales puede ser inmediata o tardía. La mayoría de los casos se diagnostican en los primeros meses. En caso de una reacción aguda inflamatoria, ésta se presenta en el postoperatorio temprano. En pacientes asintomáticos el diagnóstico se hace con los estudios imagenológicos de control generando muchas veces confusión y falsos diagnósticos ${ }^{5,11}$.

Imagenológicamente la TAC puede ser muy confusa ya que estos materiales pueden no ser radiopacos. En la resonancia magnética, éstas pueden ser de baja señal por el poco contenido de proteínas. En algunos casos, se observa hipercaptación del contraste sugiriendo la presencia de un absceso, recurrencia de una lesión tumoral, radionecrosis, encefalomalacia o infarto cerebral. Los gossypibomas se deben considerar en el diagnóstico diferencial de aquellos pacientes a los que se les ha realizado una craneotomía previamente. $(\text { Tabla } 1)^{57,9,11}$.

Microscópicamente se observan varias proporciones de actividad celular inflamatoria tanto aguda como crónica. Además se pueden encontrar depósitos de colágeno, proliferación vascular inflamatoria, fibrosis, y degeneración del material extraño ${ }^{11}$.

Cuando se utiliza un material hemostático, éste desencadena una respuesta inflamatoria local y finalmente se reabsorbe. La mayoría de estos casos son asintomáticos. Sin embargo, hay ocasiones en las cuales se produce una 
Tabla 1

Diagnósticos diferenciales de gossypibomas intracraneales

- Hematoma

- Absceso

- Tumor cerebral

- Glioma

- Recurrencia de tumor cerebral

- Metástasis

- Radionecrosis

- Infarto cerebral

- Áreas de encefalomalacia

respuesta inflamatoria exagerada alrededor de este material hemostático produciendo un efecto de masa ${ }^{2,3}$. Se ha visto que el colágeno microfibrilar se asocia con infiltrado eosinofílico, lo que no sucede con otros agentes hemostáticos. Este infiltrado es comparable con casos de colitis serosa y a reacciones alérgicas sistémicas. La reacción inflamatoria de este material puede semejarse a un glioma de alto grado incluyendo los glioblastomas multiformes por lo cual debe incluirse dentro del diagnóstico diferencial ${ }^{11}$.

El empleo de material hemostático reabsorbible es rutinario en la mayoría de los procedimientos neuroquirúrgicos. La reacción inflamatoria puede ser tan severa que puede llegar a producir efecto de masa a nivel local. Todos los agentes hemostáticos pueden causar gossypibomas. Se recomienda el retiro de estos materiales después de lograr la hemostasia o en su defecto dejar la menor cantidad posible al finalizar la cirugía ${ }^{11}$.

\section{Gossypiboma espinal}

La mayoría de los gossypibomas a nivel espinal son secundarios a gasas retenidas en el espacio paraespinal. Hoy en día es menos frecuente esta patología por el control estricto del conteo quirúrgico y la presencia de material radiopaco en las gasas que ayudan a diagnosticarlo prontamente. Hasta la fecha hay 32 casos de gossypibomas espinales publicados, de los cuales 30 son secundarios a gasas y 2 por cotonoides. La mayoría de los casos reportados corresponden a discectomías lumbares ${ }^{1,4,11}$.

El diagnóstico de los gossypibomas espinales no es fácil. Pueden ser asintomáticos o presentarse como una enfermedad inflamatoria sistémica severa. La encapsulación y la adhesión a los músculos y el tejido óseo son características comunes de los gossypibomas. Estos se presentan generalmente como una masa. Los textilomas espinales se comportan de manera silente siendo diagnosticados en forma incidental hasta 16 años después, similar a nuestro caso ${ }^{1,4}$. En caso de reacción aguda exudativa, éstos se diagnostican por la respuesta inflamatoria local asociada
Tabla 2

Diagnósticos diferenciales de gossypiboma espinal

- Hematoma

- Absceso

- Tumor

- Shwannoma

- Neurofibroma

- Histiocitoma maligno

- Fibrosarcoma

- Rabdomiosarcoma

- Leiomiosarcoma

- Neurofibromatosis

a síntomas inflamatorios sistémicos ${ }^{4}$.

Imagenológicamente los textilomas se caracterizan por la reacción inflamatoria que desencadenan. En la TAC se ven como masas de baja densidad que presentan una cápsula hiperdensa al administrar medio de contraste. Un signo característico en la TAC, es el patrón esponjoso formado por burbujas de aire. Este signo puede no verse en los casos crónicos por la absorción del aire ${ }^{1,6,12,15}$. La resonancia magnética muestra una estructura heterogénea que puede tener señales variables dependiendo de la cantidad de proteínas y de fluido que ésta contenga. Estas lesiones son hipointensas en el T1 e hiperintensas en el T2. La cápsula es hipointensa en ambas señales. En caso de que el centro de la lesión sea hiperintenso en el T1 y en el T2, indica que la lesión tiene un alto contenido hemático. Si el centro es hipo-intenso en ambas señales, sugiere que el centro contiene material textil con poco líquido. Al inyectar contraste, la periferia de la lesión se torna híper-intensa; el centro de la lesión no capta el contraste 4 .

Macroscópicamente son lesiones encapsuladas con forma arriñonada o redondeada. La superficie externa de las lesiones es lisa y amarillenta. Histopatológicamente se encuentra el material textil envuelto por una cápsula fibrosa. Pueden contener un área necrótica en el centro. Microscópicamente se encuentran células inflamatorias no específicas con histiocitos circunscritos por tejido granular y fibroso ${ }^{4}$.

Los gossypibomas se deben incluir como diagnóstico diferencial en aquellos pacientes con masas paravertebrales y que hayan sido intervenidos previamente en ese nivel. Los diagnósticos diferenciales varían desde abscesos hasta tumores. (Tabla 2) $)^{1,4}$.

Cabe resaltar las implicaciones médico legales en las que puede verse envuelto el cirujano, desde demandas penales y civiles hasta la suspensión de su tarjeta profesional. Es por esto que deben tomarse estrictas medidas durante el acto quirúrgico dentro de la cuales se encuentran entre otras, el conteo minucioso de compresas, gasas 
y cotonoides, involucrar a las enfermeras y auxiliares de salas anotando el número de gasas, compresas y afines utilizadas. En caso de dudas se debe realizar siempre un estudio radiológico intraoperatorio ${ }^{1,17}$.

\section{Conclusión}

El gossypiboma es una patología benigna que se debe tener en cuenta cuando nos enfrentamos a una masa intracerebral o espinal en un paciente previamente operado. La sospecha clínica es fundamental y los estudios imagenológicos nos pueden ayudar a realizar el diagnóstico. Casi siempre el diagnóstico se confirma en los estudios de patología. La mejor forma de prevenir estas lesiones es haciendo un recuento exhaustivo de los materiales utilizados en cirugía y emplear agentes hemostáticos en forma cuidadosa.

\section{Bibliografía}

1. Aydogan, M., Mirzanli, C., Ganiyusufoglu, K., Tezer, M., Ozturk, I.: A 13-year-old textiloma (gossypiboma) after discectomy for lumbar disc herniation: a case report and review of the literatura. The Spine Journal 2007; 7: 618621.

2. Barbolt, T.A., Odin, M., Leger, M., Kangas, L.: Pre-clinical subdural tissue reactionand absorption study of absorbable hemostatic devices. Neurol Res. 2001; 23: 537-542.

3. Fitzgerald, L.F., Goodman, J.C.: Microscopic appearance of iatrogenic foreign bodies in neurosurgery. Clin Neuropathol 1999; $18:$ 307-310.

4. Kim, H.S., Chung, T., Suh, S.H., Kim, S.Y.: MR Imaging Findings of Paravertebral Gossypiboma. Am J Neuroradiol. 2007; 28: 709-713

5. Knowlson, G.T.G.: Gel-foam granuloma in the brain. J Neurol Neurosurg Psychiatry 1974; 37: 971-973.

6. Kopka, L., Fischer, U., Gross, A.J., et al.: CT of retained surgical sponges (textilomas): pitfalls in detection and evaluation. J Comput Assist Tomogr. 1996; 20: 919-923.

7. Kothbauer, K.F., Jallo, G.I., Siffert, J., Jimenez, E., Allen, J.C., Epstein, F.J.: Foreign body reaction to hemostatic materials mimicking recurrent brain tumor: report of three

\section{Comentario al trabajo: Gossypiboma en Neurocirugía. Descripción de un caso y revisión de la literatura de G. Chater Cure y cols.}

La presencia de materiales quirúrgicos "abandonados" (o gossypibomas, textilotas, gauzomas etc.) en el lecho intervenido es una realidad clínica que se encuentra infra- cases. J Neurosurg. 2001; 95: 503-506.

8. Lo, C.P., Hsu, C.C., Chang, T.H.: Gossypiboma of the leg: MR imaging characteristics. A case report. Korean J Radiol. 2003; 4: 191-193.

9. Mathew, J.M., Rajshekhar, V., Chandy, M.J.: MRI features of neurosurgical gossypiboma: report of two cases. Neuroradiology 1996; 38: 468-469.

10. Olnick, H.M., Weens, S.H., Rogers, J.V. Jr.: Radiologic diagnosis of retained surgical sponges. JAMA 1995; 159: 1525-1527.

11. Ribalta, T., McCutcheon, I., Neto, A., Gupt,a D., Kumar, A. J., Biddle, D., Langford, L., Bruner, J., Leeds, N., Fuller, G.: Textiloma (Gossypiboma) Mimicking Recurrent Intracranial Tumor. Arch Pathol Lab Med. 2004; 128: 749-758.

12. Sheehan, R.E., Sheppard, M.N., Hansell, D.M.: Retained intrathoracic surgical swab: CT appearances. J Thorac Imag. 2000; 15: 61.

13. Sheward, S.E., Williams, A.G. Jr, Mettler, F.A. Jr, et al.: CT appearance of a surgically retained towel (gossypiboma). J Comput Assist Tomogr. 1986; 10: 343-345.

14. Van Goethem, J.W., Parizel, P.M., Perdieus, D., Herman,s P., de Moor, J.: MR and CT imaging of paraspinal tekstiloma. J Comput Assist Tomogr. 1991; 15: 1000-1003.

15. Williams, R.G., Bragg, D.G., Nelson, J.A.: Gossypiboma: the problem of the retained surgical sponge. Radiology 1978; 129: 323-326.

16. Yamato, M., Ido, K., Izutsu, M., et al.: CT and ultrasound findings of surgically retained sponges and towels. J Comput Assist Tomogr 1987; 11: 1003-1006.

17. Zbar, P., Agrawal, A., Saeed, I. T., Utidjian, M.R.A.: Gossypiboma revisited: a case report and review of the literature. J. Coll. Surg. Edinb . 1998;43: 417-441.

Chater-Cure, G.; Fonnegra-Caballero, A.; Baldión- Elorza, A.M.; Jiménez-Hakim, E.: Gossypiboma en Neurocirugía. Descripción de un caso y revisión de la literatura. Neurocirugía 2009; 20: 44-49.

Correspondencia postal: George Chater Cure. MD, Enrique Jiménez Hakim. MD. Fundación Santa Fe de Bogotá. Av 9 \#117-20, Asociacion Médica Los Andes cons (822) Bogotá. Colombia. Email: gchater@hotmail.com,ejimenezmd@gmail.com

rreportada, probablemente porque inmediatamente evoca implicaciones medicolegales y de mala práctica ${ }^{5}$.

Tal como relatan los autores en este trabajo, los gossy- 
pibomas presentan un amplio rango de presentaciones clínicas: hallazgos en pacientes asintomáticos, retraso de la curación de heridas quirúrgicas, infecciones o reacciones inflamatorias estériles (a cuerpo extraño) ${ }^{1,2,3,5,10}$.

Aunque no hay muchos datos sobre el aspecto radiológico de estas lesiones, se debe tener en cuenta que en algunos casos no presentan marcadores radio-opacos. Incluso en presencia de éstos pueden ser pasadas por alto en la radiografía simple en un $10-30 \%$ de los $\operatorname{casos}^{4,9}$. En la TAC se pueden ver hiperdensos, con captación heterogénea de contraste y en ocasiones se puede apreciar la imagen propia de un absceso con gas en su interior. Tal como describen los autores en $\mathrm{T} 1$ suelen observarse como masas hipointensas, mientras que su aspecto en T2 depende del tiempo de evolución. En fase aguda-subaguda se aprecia un centro hipointenso rodeado de un halo hiperintenso, en tanto que con el paso del tiempo, si aparece necrosis central y formación de una cápsula fibrosa el centro puede aparecer hiperintenso y rodeado de una cápsula hipointensa ${ }^{5,7}$. Sin embargo estos datos en RM no son constantes, por lo que es importante conocer que tanto la intensidad de señal, como su patrón, como el grado y patrón de captación de contraste son variables ${ }^{8}$.

Se han descrito algunos factores de riesgo para el "abandono" de materiales quirúrgicos, como la cirugía de urgencia, el elevado índice de masa corporal del paciente o los cambios no previstos de la estrategia quirúrgica (sin poder descartar otros factores como la complejidad de la cirugía, la incorporación/salida durante la intervención de los cirujanos adjuntos en hospitales docentes, los defectos de comunicación entre el personal de quirófano, la fatiga laboral o la complejidad de la cirugía $)^{6}$.

Es fundamental la prevención a través de contajes quirúrgicos (tanto de gasas como de instrumental), que algunos autores recomiendan en todo tipo de cirugía, evitando cortar los hilos de las lentinas y revisando meticulosamente el campo quirúrgico previamente al cierre. Se estima también que la realización de controles radiológicos en cualquier paciente de alto riesgo (no sólo aquellos con contajes erróneos) presenta una relación coste/beneficio favorable. En procesos legales por esta patología se ha condenado a los cirujanos incluso en presencia de contajes completos y a pesar de reporte por el personal de enfermería del estricto cumplimiento por parte del cirujano de los estándares del procedimiento quirúrgico ${ }^{5,6}$.

El tratamiento en aquellos casos sintomáticos es la resección quirúrgica, en tanto que en los casos asintomáticos sería interesante conocer cuántos cirujanos estarían dispuestos a un seguimiento sin resección de la lesión y el cuerpo extraño, y sobre todo el tipo de información que le daríamos al paciente para seguir con esta actitud expectante.
Es posible que la mayoría de cirujanos se enfrenten a este problema en algún momento a lo largo de su práctica (tanto por pacientes propios como en segundas consultas), y el reconocimiento de esta entidad resultará fundamental para una adecuada información al paciente, el tratamiento óptimo y el manejo de las posibles implicaciones legales del caso. Teniendo en cuenta el aumento que se está produciendo de las solicitudes de "segundas opiniones", hemos de considerar que de la misma manera que valoraremos pacientes tratados previamente por otros cirujanos, los pacientes operados por nosotros buscarán también la opinión de otros médicos.

\section{Bibliografía}

1. Alander, D.H., Stauffer, E.S.: Gelfoam-induced acute quadriparesis after cervical decompression and fusion. Spine 1995; 20: 970-971.

2. Friedman, J., Whitecloud, T.S., III: Lumbar cauda equina syndrome associated with the use of gelfoam: case report. Spine 2001; 26: 485-487.

3. Gifford, R.R., Plaut, M.R., McLeary, R.D.: Retained surgical sponge following laminectomy. JAMA 1973; 223: 1040.

4. Kopka, L., Fischer, U., Gross, A.J., Funke, M., Oestmann, J.W., Grabbe, E.: CT of retained surgical sponges (textilomas): pitfalls in detection and evaluation. J Comput Assist Tomogr 1996; 20: 919-923.

5. Marquardt, G., Rettig, J., Lang, J., Seifert, V.: Retained surgical sponges, a denied neurosurgical reality? Cautionary note. Neurosurg Rev 2001; 24: 41-43.

6. McLeod, R.S., Bohnen, J.M.: Canadian Association of General Surgeons Evidence Based Reviews in Surgery. 9. Risk factors for retained foreign bodies after surgery. Can J Surg 2004; 47: 57-59.

7. Nabors, M.W., McCrary, M.E., Clemente, R.J. et al.: Identification of a retained surgical sponge using magnetic resonance imaging. Neurosurgery 1986; 18: 496-498.

8. Pérez-Núñez, A., Rivas, J.J., Gómez, P.A., Cabrera, A., Palomino, J.C., Miranda, P.: Gasas quirúrgicas retenidas tras cirugía espinal lumbar: Presentación clínica y radiológica en dos casos. Neurocirugía 2004; 15 (Supl): 72.

9 Revesz, G., Siddiqi, T.S., Buchheit, W.A., Bonitatibus, M.: Detection of retained surgical sponges. Radiology 1983; 149: 411-413.

10. Vishteh, A.G., Apostolides, P.J., Dean, B., Spetzler, R.F.: Magnetic resonance image of postcraniotomy retained cotton or rayon. Case illustration. J Neurosurg 1998; 88: 928.

A. Pérez-Nuñez Madrid 